\section{Perfil de gestantes atendidas nos períodos pré-natal e perinatal: estudo comparativo entre os serviços público e privado em Guaratinguetá, São Paulo}

\section{Pregnants profile in prenatal and perinatal stages: comparative study between public and private health services in Guaratinguetá, São Paulo}

Luiz Fernando C. Nascimento 1

${ }^{1}$ Serviço de Pediatria. Faculdade de Medicina. Universidade de Taubaté. Avenida Tiradentes, 500. Taubaté, SP, Brasil. CEP: 12.080-130. E-mail: lfcnascimento@uol.com.br

\begin{abstract}
Objectives: to compare ratios of pregnant women seen in public health clinics and private health clinics according to social, demographic and medical variables.

Methods: a cross-sectional study was performed with 590 mothers who visited the Health Clinic of Guaratinguetá, for routine vaccination of the last child.

Results: analysis of the survey demonstrated higher statistically significant ratios of mothers with a shorter period of time between pregnancies, smokers, less educated, aged 19 or less, unemployed, who had normal deliveries with less medical visits during prenatal care, with reports of urinary infection during prenatal care and who did not have the assistance of a Pediatrician at the time of delivery, whose newborns did not stay with them in the room and had low birth weights, among the mothers whose prenatal care was provided in public clinics.

Conclusions: the inclusion of one item related to prenatal care provision in the "Born Alive Report" of the Ministry of Health would enable routine analysis of the services provided to mothers in public and private clinics.
\end{abstract}

Key words Pregnancy, Services evaluation, Maternal health services, Prenatal care

\section{Resumo}

Objetivos: comparar as proporções de gestantes atendidas nos serviços de saúde público e privado segundo variáveis sociais, demográficas e médicas.

Métodos: estudo transversal realizado com 590 mães que freqüentaram o Centro de Saúde de Guaratinguetá, para vacinação de rotina do último filho.

Resultados: a análise mostrou, dentre aquelas que realizaram o pré-natal no serviço público, maior proporção de mães com menor intervalo interpartal, fumantes, mais baixa escolaridade, idade menor ou igual a 19 anos, desempregadas, que realizaram parto vaginal, menor número de consultas no pré-natal, relato de infecção urinária durante o pré-natal, sem pediatra no momento do parto, e cujos recém-nascidos não ficaram em alojamento conjunto ou tinham baixo peso ao nascer.

Conclusões: a inclusão de um item sobre o local de realização do pré-natal, na "Declaração de Nascido Vivo" do Ministério da Saúde, poderia permitir análises rotineiras sobre o atendimento às mães pelos serviços público e privado.

Palavras-chave Gravidez, Avaliação dos serviços, Serviços de saúde materna, Cuidado pré-natal 


\section{Introdução}

$\mathrm{O}$ atendimento à gestante durante o pré-natal, de acordo com a fonte pagadora do serviço, não costuma ser objeto de estudos epidemiológicos específicos ou incluído como mais uma variável no rol daquelas que costumam ser analisadas neste tipo de pesquisa. Este atendimento à gestante pode ser realizado nas Unidades Básicas de Saúde cuja fonte pagadora é Sistema Público de Saúde ou em consultórios privados, onde as consultas são pagas diretamente pelo cliente ou indiretamente, através de convênios médicos ou sistema de seguro saúde.

Mesmo a "Declaração de Nascido Vivo" do Ministério da Saúde, que contempla inúmeras variáveis relacionadas com dados maternos e do recém-nascido, não contempla a informação do local onde foi realizado o pré-natal.

As variáveis que costumam ser analisadas nos estudos epidemiológicos envolvendo as gestantes geralmente são: idade materna, número de filhos anteriores, grau de escolaridade, ganho de peso durante a gestação, número de consultas no atendimento prénatal, hipertensão arterial e sangramento na gestação, menor intervalo interpartal, tabagismo e infecção urinária.1,2

Isto visa, geralmente, o estudo do desfecho da gravidez no que diz respeito ao recém-nascido de baixo peso (RNBP) ou pré-termo (RNPT), situações que contribuem sobremaneira para a morbi-mortalidade infantil,3-5 tipo de parto6 e até mesmo aleitamento materno. ${ }^{7}$ Dessa maneira, o atendimento à gestante durante o período pré-natal pode ser determinante para o aparecimento destas condições desfavoráveis no RN.

Para a comparação entre o tipo de serviço prestador da assistência do pré-natal, conforme a fonte pagadora - se pelo sistema de saúde pública, ou pelo sistema privado - Krieger et al.,8 nos Estados Unidos, mostraram haver diferenças no perfil das gestantes atendidas pelo Medicaid, que é um programa de cobertura de saúde para as famílias de baixa renda e as atendidas pelo sistema privado. As gestantes atendidas pelo Medicaid apresentavam menor idade materna, maior proporção de fumantes na gravidez e menor intervalo interpartal, quando comparadas com as atendidas no pré-natal pelo sistema privado.

Dobie et al., 9 analisando dados sobre cuidados no pré-natal em mulheres cujas gravidezes eram de baixo risco, encontraram menor idade materna, maior paridade, menor número de consultas no prénatal e maior número de partos vaginais dentre as mães atendidas pelo Medicaid, em comparação com o atendimento pelo sistema privado. Nesta mesma linha, Kaestner10 encontrou proporção maior de RNBP, menor número de consultas no pré-natal e número maior de mães fumantes dentre as mulheres atendidas pelo Medicaid.

Também a mortalidade neonatal parece estar relacionada ao tipo de atendimento, considerando-se a realização do parto, se através do Sistema Único de Saúde (SUS) ou privado (não-SUS). Sob este enfoque, Monteiro11 concluiu que a mortalidade neonatal era 1,8 vezes maior nos hospitais vínculados ao SUS quando comparada com a mortalidade neonatal em hospitais privados ou não-SUS.

Assim, o presente trabalho tem por objetivo comparar as proporções de determinadas variáveis, características de gestantes e de recém-nascidos, segundo o local de realização do pré-natal, isto é, se a fonte pagadora é o Sistema Único de Saúde ou outras fontes.

\section{Métodos}

Trata-se de um estudo transversal, envolvendo mães que compareceram ao setor de vacinação do Centro de Saúde de Guaratinguetá, SP, Brasil, para vacinação de rotina do último filho. O Centro de Saúde é a maior unidade de vacinação da cidade, com cerca de $90 \%$ do movimento de vacinação, já que o município não conta com unidades privadas para vacinação de rotina. Isto faz com que todos os estratos sociais se valham desse serviço para vacinação; desta forma, esta amostra deve representar a população local. Guaratinguetá situa-se no Vale do Paraíba paulista, entre São Paulo e Rio de Janeiro, contando com cerca de 100.000 habitantes.

As mães, informadas dos objetivos da pesquisa, após consentimento, responderam a um questionário com conteúdo relacionado a aspectos sociais, demográficos e médicos da gestante e desta última gravidez, dados do recém-nascido e do período perinatal. Foram incluídas somente as mães que deram à luz no ano de 1998. As entrevistas eram diárias, realizadas pelo autor com todas as mães que freqüentaram este serviço e que foram convidadas a participar do estudo.

As variáveis de estudo relacionadas com aspectos sociais e demográficos foram: paridade, presença ou não de outros filhos com baixo peso, intervalo interpartal, categorizado em até 24 meses e de 25 meses e mais entre o filho anterior e este, hábito de fumar, grau e instrução materna, categorizada em até oito anos, e nove anos e mais de escolaridade, idade materna dividida em até 19 anos, 20 a 34 anos e 35 anos e mais, situação conjugal, (co-habitação com o mari- 
do ou companheiro ou vivendo só), e situação trabalhista empregada ou não.

As variáveis médicas foram: tipo de parto, se vaginal ou operatório, peso do recém-nascido, (categorizado como baixo peso aquele que nasceu com menos de $2.500 \mathrm{~g}$ ), ganho de peso nesta gestação (categorizado em classes de até $10 \mathrm{~kg}$ e $11 \mathrm{~kg}$ ou mais). Presença de sangramento, infecção urinária e história de hipertensão arterial nesta gestação foram consideradas presentes ou ausentes; as consultas foram agrupadas em até seis, e sete ou mais consultas; a presença de pediatra no momento do parto e alojamento conjunto durante a estadia no hospital foram consideradas como variáveis perinatais.

A variável dependente foi o local de atendimento no período pré-natal, se realizado em Unidades Básicas de Saúde cuja fonte pagadora é o SUS ou em consultórios privados nos quais incluem-se além do pagamento particular, os atendimentos pelos convênios médicos e seguros saúde como fontes pagadoras.

Para análise de associação entre variáveis nominais foi utilizada a técnica do qui-quadrado; para a análise de variáveis contínuas foi empregada a técnica $t$ de Student e para variáveis ordinais, a técnica de Mann-Whitney. Para se compararem as proporções de mães atendidas nos serviços público e privado, foi aplicada a técnica da Razão de Proporção (RP), sendo construídos intervalos de confiança de $95 \%$ (IC95\%). Para identificar possíveis fatores de confusão ou de interação, foi realizada a análise estratificada, segundo a instrução materna, considerada como uma variável socioeconômica. Foram criados dois estratos, um de mães com até oito anos de escolaridade e outro de mães com nove anos e mais de escolaridade. Através da análise estratificada foram obtidos os valores das Odds ratio (OR) brutas e pon- deradas e o valor do qui quadrado de interação das variáveis independentes.

A significância estatística foi $\mathrm{p}<0,05$. A amostra foi estimada, baseando-se num erro alfa de $5 \%$, erro beta de $20 \%$ e para se detectar uma razão de proporção de 1,50. Resultou daí uma amostra mínima de 525 mães. Em decorrência da não resposta a determinados itens, alguns totais foram inferiores ao número de mães entrevistadas.

Todos os dados obtidos referem-se à última gestação materna.

\section{Resultados}

Foram entrevistadas 590 mães que freqüentaram o Centro de Saúde entre os meses de maio e agosto de 1998, para vacinação de rotina de seus filhos. Este total representa cerca de $30 \%$ dos partos ocorridos anualmente em Guaratinguetá. Dentre estas mães, $380(64,4 \%)$ fizeram o pré-natal em consultórios públicos.

A idade materna média das gestantes atendidas no serviço público foi de 24,0 anos e das atendidas no serviço privado foi de 26,7 anos $(t=5,37, p<0,001)$. A idade materna média, quando a mãe estava dando à luz pela primeira vez, foi de 20,8 anos para as mães atendidas pelo serviço público e de 24,5 anos, para as mães atendidas pelo sistema privado, sendo esta diferença estatisticamente significativa ( $p<0,001)$.

A paridade entre as mães que fizeram pré-natal no serviço público foi de 2,0 filhos e das mães que fizeram pré-natal no serviço privado foi de 1,7 , (p $=0,001)$.

As variáveis sociais e demográficas estão descritas na Tabela 1 . 
Distribuição das variáveis sociais e demográficas das gestantes atendidas nos serviços público e privado, com as respectivas razões de prevalência (RP) e intervalos de confiança de 95\% (IC95\%).

\begin{tabular}{|c|c|c|c|c|c|c|c|}
\hline \multirow{2}{*}{ Variáveis sociais e demográficas } & \multicolumn{2}{|c|}{ Serviço público } & \multicolumn{2}{|c|}{ Serviço privado } & \multirow{2}{*}{$\mathbf{R P}$} & \multirow{2}{*}{ IC95\% } & \multirow{2}{*}{$p$} \\
\hline & $\mathrm{n}$ & $\%$ & $\mathrm{n}$ & $\%$ & & & \\
\hline \multicolumn{8}{|l|}{ Outros filhos com baixo peso (284) * } \\
\hline Sim & 39 & 19,8 & 10 & 11,5 & 1,72 & $0,90-3,29$ & 0,09 \\
\hline Não & 158 & 80,2 & 77 & 88,5 & & & \\
\hline \multicolumn{8}{|l|}{ Intervalo interpartal (298) * } \\
\hline Até 24 meses & 102 & 48,8 & 30 & 33,7 & 1,45 & $1,05-2,00$ & 0,02 \\
\hline$>24$ meses & 107 & 51,2 & 59 & 66,3 & & & \\
\hline \multicolumn{8}{|l|}{ Fumante (589) } \\
\hline $\operatorname{Sim}$ & 87 & 22,9 & 21 & 10,1 & 2,28 & $1,46-3,56$ & $<0,001$ \\
\hline Não & 293 & 77,1 & 188 & 89,9 & & & \\
\hline \multicolumn{8}{|l|}{ Instrução (590) } \\
\hline Até 8 anos ** & 289 & 76,1 & 59 & 28,1 & 2,71 & $2,17-3,39$ & $<0,001$ \\
\hline 9 anos e mais & 91 & 23,9 & 151 & 71,9 & & & \\
\hline \multicolumn{8}{|l|}{ Idade materna (590) } \\
\hline Até 19 anos & 94 & 24,7 & 27 & 12,9 & 1,81 & $1,23-2,67$ & $<0,001$ \\
\hline $20-34$ anos ** & 263 & 69,2 & 159 & 75,7 & & & \\
\hline 35 anos e mais & 23 & 6,1 & 24 & 11,4 & 0,53 & $0,31-0,92$ & 0,02 \\
\hline \multicolumn{8}{|l|}{ Empregada (590) } \\
\hline Sim & 62 & 16,3 & 75 & 35,7 & 0,46 & $0,34-0,61$ & $<0,001$ \\
\hline Não & 318 & 83,7 & 135 & 64,3 & & & \\
\hline \multicolumn{8}{|l|}{ Situação conjugal (586) } \\
\hline Vive maritalmente & 303 & 79,7 & 177 & 85,5 & 0,93 & $0,87-1,01$ & 0,08 \\
\hline Vive só & 76 & 20,3 & 30 & 14,5 & & & \\
\hline
\end{tabular}

Os números entre parênteses referem-se ao total de respostas para a variável; * Referem-se a mães com mais de um filho; * Categoria de referência. RP = razão de prevalência.

Pode-se observar que houve maior proporção de mães com menor intervalo interpartal, fumantes, menor escolaridade, idade igual ou menor que 19 anos e sem emprego, no conjunto de mães que realizaram o pré-natal através do serviço público, quando comparado com aquelas mães que realizaram o pré-natal no serviço privado. Para a variável presença de outros filhos com baixo peso, há uma maior prevalência entre as mães que fizeram o pré-natal no serviço público, porém sem significância estatística. Para a situação conjugal a RP é próxima de um.

Quando se categoriza o peso ao nascer, o baixo peso é quase duas vezes mais prevalente entre os filhos cujas mães freqüentaram o serviço público (Tabela 2). Para as gestantes atendidas no serviço público, quando comparadas com as mães atendidas através do serviço privado, foram observadas freqüências maiores de: parto vaginal, menor número de consultas no pré-natal, menor ganho de peso, maior freqüência de infecção urinária e freqüências menores de presença de pediatra na sala, no momento do parto, e de alojamento conjunto.

Das mães que fizeram o pré-natal no serviço público, 70,4\% foram consultadas sete vezes ou mais e das que fizeram o pré-natal no serviço privado este percentual foi de $83,7 \%$. (Tabela 2). 
Distribuição das variáveis perinatais e médicas das gestantes atendidas nos serviços público e privado, com as respectivas razões de prevalência (RP) e intervalos de confiança de 95\% (IC95\%).

\begin{tabular}{|c|c|c|c|c|c|c|c|}
\hline \multirow{2}{*}{ Variáveis perinatais e medicas } & \multicolumn{2}{|c|}{ Serviço público } & \multicolumn{2}{|c|}{ Serviço privado } & \multirow{2}{*}{$\mathbf{R P}$} & \multirow{2}{*}{$\mathrm{IC} 95 \%$} & \multirow{2}{*}{$p$} \\
\hline & $\mathrm{n}$ & $\%$ & $\mathrm{n}$ & $\%$ & & & \\
\hline \multicolumn{8}{|l|}{ Tipo de parto $(590)$} \\
\hline Vaginal & 157 & 41,3 & 29 & 13,8 & 2,99 & $2,08-4,25$ & $<0,001$ \\
\hline Cesáreo & 223 & 58,7 & 181 & 86,2 & & & \\
\hline \multicolumn{8}{|l|}{ Consultas (585) } \\
\hline até 6 & 112 & 29,6 & 34 & 16,3 & 1,82 & $1,30-2,58$ & $<0,001$ \\
\hline 7 e mais & 264 & 70,4 & 175 & 83,7 & & & \\
\hline \multicolumn{8}{|l|}{ Ganho de peso (536) } \\
\hline até $10 \mathrm{~kg}$ & 191 & 56,3 & 91 & 46,2 & 1,22 & $1,02-1,46$ & 0,02 \\
\hline $11 \mathrm{~kg}$ e mais & 148 & 43,7 & 106 & 53,8 & & & \\
\hline \multicolumn{8}{|l|}{ Sangramento (584) } \\
\hline Presente & 43 & 11,5 & 26 & 12,4 & 0,93 & $0,59-1,47$ & 0,75 \\
\hline Ausente & 331 & 88,5 & 184 & 87,6 & & & \\
\hline \multicolumn{8}{|l|}{ Infecção urinária (581) } \\
\hline Presente & 74 & 19,9 & 24 & 11,4 & 1,75 & $1,14-2,68$ & 0,01 \\
\hline Ausente & 297 & 80,1 & 186 & 88,6 & & & \\
\hline \multicolumn{8}{|l|}{ Hipertensão arterial (584) } \\
\hline Presente & 58 & 15,5 & 26 & 12,4 & 1,25 & $0,81-1,93$ & 0,30 \\
\hline Ausente & 316 & 84,5 & 184 & 87,6 & & & \\
\hline \multicolumn{8}{|l|}{ Pediatra na sala (562) } \\
\hline Presente & 272 & 76,2 & 191 & 93,2 & 0,82 & $0,76-0,88$ & $<0,001$ \\
\hline Ausente & 85 & 23,8 & 14 & 6,8 & & & \\
\hline \multicolumn{8}{|l|}{ Alojamento conjunto (579) } \\
\hline Presente & 285 & 76,8 & 173 & 83,2 & 0,92 & $0,85-1,00$ & 0,06 \\
\hline Ausente & 86 & 23,2 & 35 & 16,8 & & & \\
\hline Baixo peso ao nascer (587) & 41 & 10,9 & 12 & 5,7 & 1,89 & $1,02-3,51$ & 0,04 \\
\hline Peso normal & 337 & 89,1 & 197 & 94,3 & & & \\
\hline
\end{tabular}

Os números entre parênteses referem-se ao total de respostas para a variável. RP = razão de prevalência.

Quando se estratifica a análise segundo a escolaridade materna, observa-se que o grau de instrução materna é variável modificadora de efeito (interação) para a variável infecção urinária (qui-quadrado = $5,09, \mathrm{p}=0,026)$ sendo que a prevalência desta doença é quatro vezes maior nas gestantes que fizeram o prénatal no serviço público com grau de instrução até oito anos do que naquelas com maior escolaridade (até oito anos: hospitais públicos 21,1\%; hospitais privados $5,1 \%$. Mais de oito anos: hospitais públicos $14,2 \%$; hospitais privados $13,9 \%$. Por outro lado, a instrução materna, parece ser variável de confusão para a variável fumante, com $\mathrm{OR}_{\text {bruta }}=2,66 \mathrm{e}$ $\mathrm{OR}_{\mathrm{MH}}=1,69$ e para ganho de peso na gestação, $\mathrm{OR}_{\text {bruta }}=1,50$ e $\mathrm{OR}_{\mathrm{MH}}=1,21$. 


\section{Discussão}

Apesar de não serem comuns estudos abordando este tema, foram confirmadas neste trabalho diferenças entre as gestantes, segundo o local de atendimento durante o pré-natal, bem como em determinadas características do $\mathrm{RN}$ e também em variáveis do período perinatal como, presença do pediatra no momento do parto e alojamento conjunto. A importância da avaliação da qualidade do atendimento à gestante também se prende ao esforço de reduzir os valores das taxas de mortalidade materna, que é de 55,7 óbitos maternos/100.000 nascidos vivos no estado de São Paulo e também de mortalidade perinatal, que é de cerca de 23,6 óbitos/1.000 nascidos vivos. 12

A gestante atendida no serviço público geralmente é aquela que não pode pagar pelo serviço profissional, sendo a consulta paga pelo Sistema Único de Saúde. Esta camada da sociedade costuma ter um menor poder aquisitivo e maior dificuldade para chegar aos serviços de saúde tanto por residirem em locais afastados como por falta de orientação sobre a importância do acompanhamento pré-natal.

Assim, pode-se observar que há uma proporção maior de mães, quando o atendimento foi realizado no serviço público, que tinham completado, no máximo, o primeiro grau, quando comparada com os segundo e terceiro graus. Houve menor proporção de mães empregadas dentre aquelas atendidas no serviço público. Situação semelhante foi descrita por Cesar e Tanaka13 que encontraram uma diminuição progressiva do atendimento público, ao pré-natal e também ao parto, com o aumento da escolaridade materna, em um estudo realizado na região da Grande São Paulo.

Outro dado, coincidente com o observado por Krieger et al.,8 em que pesem as diferenças entre os Sistemas Medicaid e Único de Saúde, foi a idade materna média menor dentre as atendidas no serviço público; mesmo estratificando pela escolaridade, as idades maternas médias se mantêm menores naquelas atendidas pelo serviço público. Possivelmente as mães atendidas no serviço privado têm melhor orientação com referência aos métodos anticoncepcionais ou estão no pleno exercício profissional, desta forma, vindo a engravidar mais tardiamente como visto. No estado de São Paulo, a taxa de escolaridade no sexo feminino, no ano de 1998, foi 48,2\% de mulheres com mais de oito anos de estudo e a taxa encontrada neste estudo foi $41,1 \%$. Da mesma forma, os achados deste estudo vão ao encontro, também, dos resultados obtidos quanto ao fumo.

O menor intervalo interpartal, observado entre esta gravidez e a anterior, dentre as mães atendidas pelo sistema público de saúde, pode ser reflexo de menor acesso a esse serviço, com menos orientação para a anticoncepção, somado a uma menor escolaridade. $^{2}$

Menor número de consultas no pré-natal e maior paridade também coincidem com os achados de Dobie et al. ${ }^{9}$ Esses dois fatores parecem estar ligados e podem decorrer do desconhecimento da importância do pré-natal e de métodos anticonceptivos bem como de um desinteresse das mulheres com menor grau de instrução. Nesta casuística, 75,0\% das mães informaram ter realizado mais de sete consultas no pré-natal, sendo que as atendidas no serviço privado foram $83,7 \%$ e no serviço público, 70,4\%. Dados do Ministério da Saúde relativos ao número de consultas, no estado de São Paulo no ano de 1998, mostram valores de 59,2\%; assim a cobertura do pré-natal, nesta cidade, mostrou-se acima dos dados do Estado. 14

Dentre as variáveis médicas, o parto vaginal foi o que apresentou maior proporção; esse dado é semelhante aos mostrados por Silva et al., 6 em São Luiz, MA, onde a chance de se realizar cesareana era cinco vezes maior nas gestantes atendidas no serviço privado, quando comparada com a gestante atendida pelo serviço público. Haidar et al. ${ }^{2}$ mostraram a forte associação entre parto cesáreo e maior escolaridade materna, podendo explicar esse achado dentre as atendidas no serviço privado. Todos os partos deste estudo foram hospitalares, assim como o foram 99,3\% dos partos realizados no estado de São Paulo em 1998.14

O baixo peso ao nascer também foi observado com frequiência duas vezes maior entre as mães atendidas pelo sistema público, dados coincidentes com os de Silva et al. ${ }^{6}$ o que é preocupante dada a maior morbimortalidade nesse grupo de crianças. ${ }^{3}$

O menor número de consultas no pré-natal pode estar associado a um início mais tardio, ou a dificuldades de acesso aos serviços de saúde. Tal situação pode dificultar o diagnóstico e tratamento de determinadas situações, como menor ganho de peso gestacional, que é um fator associado ao baixo peso ao nascer. $15 \mathrm{O}$ menor número de consultas no prénatal também está associado, como fator de risco, ao nascimento pré-termo. 5 O menor número de consultas no pré-natal pode comprometer o papel educativo que poderia ser desenvolvido com as gestantes, gerando dificuldades na conscientização dessas para o alojamento conjunto, que teve menor proporção dentre as gestantes atendidas pelo serviço público, com significância estatística. Como o alojamento conjunto tem papel importante para o aleitamento materno $^{7}$ a falta daquele pode diminuir a prevalência 
do aleitamento.

A maior prevalência de infecção urinária dentre as gestantes atendidas no serviço público é outro dado preocupante, dada à importância dessa situação para o nascimento pré-termo e baixo peso.5,15 Tal fato poderia ser controlado com maior número de consultas.

Outra situação observada neste estudo foi a menor freqüência da presença do pediatra na sala de parto, no momento do nascimento. Essa ausência pode ser determinante para um atendimento de pior qualidade para o recém-nascido frente a uma situação de emergência, com possível comprometimento na vida futura. É interessante lembrar que o Sistema Único de Saúde remunera a possibilidade de atendimento do pediatra na sala de parto.

Não foi possível determinar, e não era este o objetivo do estudo, se as situações mais prevalentes nas gestantes atendidas pelo serviço público são decorrentes da forma e da qualidade do atendimento, ou se são inerentes à própria gestante, como a situação nutricional e educacional entre outras. A análise estratificada mostrou o papel importante da instrução materna quanto a determinadas situações como o tabagismo, o ganho de peso na gestação e a presença de infecção urinária.

\section{Referências}

1. Solla JJSP, Pereira RAG, Medina MG, Pinto LLS. Análisis multifactorial de los factores de riesgo de bajo peso al nascer en Salvador, Bahia. Rev Panam Salud Publica 1987; 2: 1-6.

2. Haidar FH, Oliveira UF, Nascimento LFC. Escolaridade materna: correlação com os indicadores obstétricos. Cad Saúde Pública 2001; 17: 1025-30.

3. McKormick MC. The contribuiton of low birth weight to infent mortality and childhood morbidity. N Engl J Med 1985; 85: 806-11.

4. Martius JÁ, Steck T, Oehler MK, Wulf KH. Risk factors associated with preterm $(<37+0$ weeks $)$ and early preterm birth $(<32+0$ weelks $)$ : univariate and multivariate analysis of 106345 singleton births from the 1994 statewide perinatal survey of Bavaria. Eur J Obstet Gynecol 1998; 80: 183-9.

5. Nascimento LFC. Epidemiology of preterms in Southeast Brazil: a hospital-based study. Rev Bras Saúde Matern Infant 2001; 1: 263-8.

6. Silva AAM, Lamy-Filho F, Alves MTSSB, Coimbra LC, Bettiol H, Barbieri MA. Risk factors for low birthweight in North-East Brazil: the role of caesarean section. Paediatr Perinat Epidemiol 2001; 15: 257-64.
Por outro lado, é importante lembrar que é comum os mesmos profissionais atenderem consultas de pré-natal no serviço público e também no privado, mas este estudo não discriminou essa situação. Outra limitação é que este perfil talvez possa ser encontrado em cidades com características semelhantes de população e de oferta de serviços médicos.

O tamanho da amostra mostrou-se satisfatório, tendo sido entrevistado um número acima do valor sugerido. Talvez possa ter ocorrido viés de seleção, apesar de o local onde foram realizadas as entrevistas ser o maior e com o maior movimento de vacinação da cidade. Em virtude da inexistência de centros privados para vacinação de rotina, isto é, aquelas que constam do calendário vacinal, algumas mães podem ter procurado tais serviços em outras cidades próximas, mas este número é muito pequeno. Não houve recusa por parte das mães em responderem ao questionário. Desta forma esse viés foi minimizado.

Assim, foram encontradas diferenças estatisticamente significativas no perfil das gestantes atendidas nos serviços públicos e privados. A inclusão do item local onde foi realizado o pré-natal, na Declaração de Nascido Vivo do Ministério da Saúde, poderia tornar a análise mais abrangente e realizada de uma maneira rotineira, como forma de avaliação, tanto do serviço privado como do serviço público.

7. Caldeira AP, Goulart EMA. A situação do aleitamento materno em Montes Claro. J Pediatr [Rio de Janeiro] 2001; 76: 55-64.

8. Krieger JW, Connell FA, Logerfo JP. Medicaid prenatal care: a comparasion of use and outcomes in fee-for-service and managed care. Am J Public Health 1992; 82: 185-90.

9. Dobie S, Hart LG, Fordyce M, Andrilla CHA, Rosenblatt RA. Obstetric care and payment source: do low-risk medicaid women get less care? Am J Public Health 1998; 88: 51-6.

10. Kaestner R. Health insurance, the quantity and quality of prenatal care, and infance health. Inquiry 1999; 36: 16275.

11. Monteiro CV. Mortalidade neonatal: estudo de casocontrole no município de São Paulo, 1995 [dissertação mestrado]. São Paulo: Faculdade de Saúde Pública da Universidade de São Paulo; 2000.

12. Ministério da Saúde. Taxas de mortalidade. Disponível em: 〈http://tabnet.datasus.gov.br/cgi/idb2001/c03.def $>$ [2002 out 31].

13. Cesar CLG, Tanaka OY. Inquérito domiciliar como instrumento de avaliação de serviço de saúde: um estudo de 
caso na região sudoeste da área metropolitana de São Paulo, 1989-1990. Cad Saúde Pública 1996; 12 Supl 2: 59-70.

14. Ministério da Saúde. Proporção de gestantes com acompanhamento pré-natal e partos hospitalares. Disponível em: $\quad \leq$ http://tabnet.datasus.gov.br/cgi/tabcgi.exeidb2001/f06.def $>$ [2002 out 31].
15. Horta BL, Barros FC, Halpern R, Victora CG. Baixo peso ao nascer em duas coortes de base populacional no sul do Brasil. Cad Saúde Pública 1996; 12 Supl. 1: 27-31.

Recebido em 4 de fevereiro de 2003

Versão final reapresentada em 16 de abril de 2003

Aprovado em 5 de maio de 2003 